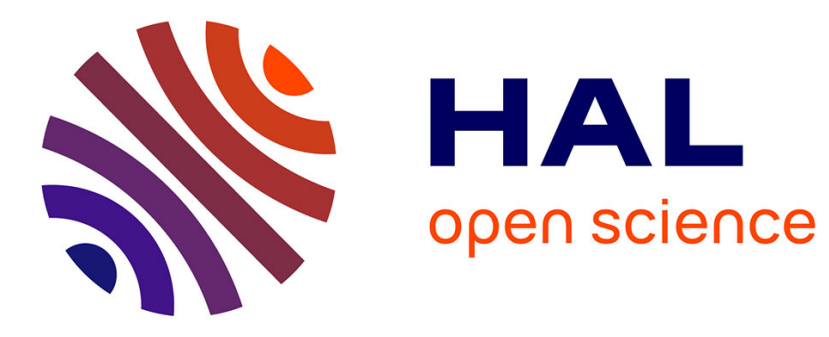

\title{
Subcritical open channel flows in four branch intersections
}

\author{
Nicolas Riviere, G. Travin, Richard J. Perkins
}

\section{To cite this version:}

Nicolas Riviere, G. Travin, Richard J. Perkins. Subcritical open channel flows in four branch intersections. Water Resources Research, 2011, 47 (10), pp.W10517. 10.1029/2011WR010504 . hal00691087

\section{HAL Id: hal-00691087 \\ https://hal.science/hal-00691087}

Submitted on 18 Apr 2019

HAL is a multi-disciplinary open access archive for the deposit and dissemination of scientific research documents, whether they are published or not. The documents may come from teaching and research institutions in France or abroad, or from public or private research centers.
L'archive ouverte pluridisciplinaire HAL, est destinée au dépôt et à la diffusion de documents scientifiques de niveau recherche, publiés ou non, émanant des établissements d'enseignement et de recherche français ou étrangers, des laboratoires publics ou privés. 


\title{
Subcritical open channel flows in four branch intersections
}

\author{
N. Rivière, ${ }^{1}$ G. Travin, ${ }^{1}$ and R. J. Perkins ${ }^{2}$ \\ Received 4 February 2011; revised 22 July 2011; accepted 7 September 2011; published 18 October 2011.
}

[1] Subcritical flow in an intersection composed of four similar orthogonal channels has been studied experimentally in a configuration with two inflows and two outflows for a wide range of experimental conditions. The results have been used to develop a relationship between the incoming flow rates and the flow distribution in the two outlet channels, based on the conservation of discharge and momentum in the intersection, and suitable stagedischarge relationships for the downstream controls in the outflow channels. A final equation is provided by an empirical correlation for the outflow in one of the channels, based on the experimental data obtained from these experiments; this correlation agrees with all the available data to within $\pm 5 \%$. It is shown how the resulting set of equations can be used to compute the discharge distribution in any similar intersection, given the incoming flow rates and some form of stage-discharge relationship for the outlet conditions.

Citation: Rivière, N., G. Travin, and R. J. Perkins (2011), Subcritical open channel flows in four branch intersections, Water Resour. Res., 47, W10517, doi:10.1029/2011WR010504.

\section{Introduction}

[2] During severe flooding of urban areas, the water in the streets can reach a depth of a meter or more, resulting in important economic losses, and possible loss of life. The prediction of flood propagation in urban areas is required in order to design suitable protection (possibly by directing the flood waters to previously identified "sacrificial" zones) and to develop realistic emergency planning. Geographical Information Systems now provide detailed descriptions of street geometry for most urban areas, so it is reasonable to envisage investigating a wide range of different scenarios using numerical simulations based on the St. Venant equations [Inoue et al., 2000; Calenda et al., 2003; Haider et al., 2003; Mignot et al.,2006; El Kadi Abderrezzak et al., 2009]. In general, the 1-D St. Venant equations provide a reasonable model for the flow in individual streets, but they are not well-suited to model street intersections where the flow is necessarily strongly three dimensional, and where the slope of the free surface can be significant. Unfortunately for these numerical simulations, street intersections often play a crucial role in determining how the incoming flow is distributed between the outlet channels. There have been few experimental studies of flow in open channel intersections, and most published studies have concerned flow distribution in networks of irrigation channels, where the intersection is usually formed by the intersection of three channels (in the form of a $\mathrm{T}$ or a Y), and where the flow regime is subcritical. These intersections function either as a convergence, two upstream reaches feeding one downstream reach, or as a bifurcation, one upstream reach feeding two downstream reaches. Although this paper

\footnotetext{
${ }^{1}$ Laboratoire de Mécanique des Fluides et d'Acoustique (LMFA), CNRS UMR5509, Université de Lyon, F-69621, Villeurbanne, France.

${ }^{2}$ Laboratoire de Mécanique des Fluides et d'Acoustique (LMFA), CNRS UMR5509, Université de Lyon, F-69134, Ecully, France.
}

Copyright 2011 by the American Geophysical Union. 0043-1397/11/2011WR010504 addresses the problem of subcritical flow in four branch intersections, some of the ideas that are used to model the flow are based on results obtained for three branch intersections, so we begin with a review of current research on that configuration.

\subsection{Three Branch Confluence}

[3] The general characteristics of subcritical flow merging in a three branch $\mathrm{T}$ junction were investigated experimentally by Weber et al. [2001], and numerically by Huang et al. [2002]. These studies showed that a three dimensional recirculating region develops downstream of the junction, in the axial branch; the extent of this region varies from the bed to the surface, and has been the subject of several investigations [Best and Reid, 1984; Hsu et al., 1998; Gurram et al., 1997; Hager, 1999]. Various 1-D models have been developed to link the flow distribution and the water depths for steady subcritical flow in different configurations of a three branch junction. Most of these models assume that the depths are identical in the two inlet channels, just upstream of the junction [e.g., Taylor, 1944; Webber and Greated,1966; Ramamurthy and Satish, 1988; Gurram et al., 1997]. This assumption has been confirmed by experiments in which the ratio of the depths in the main and branch channels entering the intersection is close to unity $(-5 \%,+2 \%$ for Hsu et al. [1998]; $1.4 \%$ for Weber et al. [2001]). If the flow is subcritical everywhere, the depth in the downstream channel is determined by the exit condition. It only remains, therefore, to compute the depths in the two upstream channels [Sridharan and LakshmanaRao, 1966]. This can be done by applying a momentum balance equation to the junction flow, as described by the previously cited authors, for example. Shabayek et al. [2002] derived a model which does not rely on the assumption of equal depths in the upstream channels but requires the solving of more complex equation. Two of the flow conditions that have been investigated in three branch confluence flows are beyond the scope of this study; the first is when the recirculating zone downstream of the junction 
causes a contraction in the channel section, provoking transition to supercritical flow, as reported by Ramamurthy et al. [1988] and Hager [1989]. The second corresponds to two supercritical incoming flows; one or more hydraulic jumps form in the junction because of the mutual deflection of the two incoming streams, producing a variety of different flow configurations, depending on the positions of the jumps, which can be either straight or oblique [Gisonni and Hager, 2002; Mignot et al., 2008b].

\subsection{Three Branch Bifurcations}

[4] The general characteristics of steady subcritical flow in a three branch bifurcation were described by Neary and Odgaard [1993]. In this case a three-dimensional recirculating region develops in the lateral branch [Neary and Odgaard, 1993; Neary et al., 1999; Ramamurthy et al., 2007; Li and Zeng, 2009a, 2009b]. The principal difference with the flow in a junction is that the distribution of the incoming flow between the two outgoing channels is unknown, and since the total flow is conserved (and known) this introduces one extra unknown quantity into the problem; a model based solely on a momentum balance of the flow in the bifurcation cannot therefore provide a solution of the problem [Taylor, 1944]. However, this approach can be used to express the flow distribution in terms of another variable which characterizes the flow, such as the ratio of Froude numbers upstream and downstream of the bifurcation [Law and Reynolds, 1966], or the ratio of the depths upstream and downstream of the bifurcation [Ramamurthy et al., 1990; Hsu et al., 2002]. An additional equation is still required to close the system and provide a full solution; one possible approach would be to use an equation based on the conservation of energy, with empirical coefficients to account for energy dissipation in the bifurcation, but these losses are so low that they are difficult to measure accurately [e.g., Hager, 1992] and cannot be accounted for by simple coefficients [Lipeme-Kouyi et al., 2010]. There have been attempts to exploit the results from other, similar flows, flow in a channel bend for Neary and Odgaard [1993] and flow in the T bifurcation of a closed conduit for Barkdoll et al. [1998], but the analogy between these flows is not sufficiently close (because of the three-dimensional nature of the flow, and the influence of variations in the depth of the flow) for this to be useful. Kesserwani et al. [2010] adopted a different approach, with some success, by exploiting an analogy with flow over side weirs with zero height. Dimensional analysis provides another possible approach for deriving an additional equation. Nougaro and Boyer [1974] provide a diagram giving the discharge ratio valid over a wide range of Froude numbers and intersection angles for free-outlet conditions. However, their results are strongly dependent on the downstream conditions [Nougaro et al., 1975]. In fact, several different flow regimes can occur, depending on the formation of hydraulic jumps, free surface waves, and other flow transitions generated by the bifurcation [Law and Reynolds, 1966]. These different regimes can be classified into three categories: subcritical flow everywhere, transition to supercritical flow in the main outlet channel, and subcritical flow everywhere except in the side branch where the contraction of the flow creates a region of choked flow. Several authors have focused on this latter case because the existence of choked flow $(F r=1)$ reduces the number of variables and limits the scope of the problem. The approach based on dimensional analysis provides empirical relationships for the flow distribution [Krishnappa and Seetharamiah, 1963; LakshmanaRao and Sridharan, 1967; Ramamurthy and Satish, 1988]. Finally, Rajaratnam [1962] showed that when the flow in the upstream branch is supercritical, the axial velocity in the intersection can be considered as constant, and this can be used to compute the distribution of the flow between the branches [Rivière and Perkins, 2004].

\subsection{Four Branch Intersections}

[5] The case of two incoming supercritical flows is beyond the scope of this paper. It has been studied experimentally by Nania et al. [2004] and Mignot et al. [2008b], numerically modeled by Mignot et al. [2008a] and analyticaly by Mignot et al. [2011]. The case of two incoming subcritical flows, with horizontal channels, was investigated experimentally and numerically by Rivière et al. [2006] but the authors were unable to develop an analytical model for the flow distribution.

[6] Two main conclusions emerge from this analysis. First, although the most common basic configuration in urban areas is the intersection consisting of four streets meeting orthogonally, most of the studies of subcritical flow in intersections have concerned three branch intersections. Second, for those configurations in which the outgoing flow is distributed between two channels, an analytical model for the flow distribution is only available for the case in which a critical section occurs in the side channel; even if the four branch intersection is considered as the superposition of a confluence and a bifurcation, it has not proved possible to derive a solution for the flow distribution.

[7] The work described in this article concerns an experimental investigation of subcritical flow in a four branch intersection, with two inlet and two outlet channels. There are three main objectives. First, the experiments will provide valuable insights into the different physical phenomena that occur in the flow. Second, the results of the experiments provide an empirical correlation for the flow distribution which complements existing one-dimensional models for this configuration. Finally, the data will be useful in validating 2-D and 3-D numerical simulations of the same flow, and this validation will be essential if the models are then to be used to investigate more complicated geometries or flow conditions. In the following section 1.4 we describe the problem in detail, the physical parameters that characterize it, and the experimental conditions. In section 2 we derive the equations necessary to define the flow in the experimental configuration, and we show how these can be generalized to any set of boundary conditions likely to be encountered in practice. In section 3 we extend existing models for a three branch intersection to our four branch configuration, based on a momentum balance, to relate the flow distribution to the depths in the channels. Finally, in section 4, we use dimensional analysis to close the problem with an empirical correlation for the flow distribution.

\subsection{The General Problem}

[8] The geometrical configuration is shown in Figure 1a; the intersection is formed from four identical canals of width $b$ meeting at right angles; the intersection itself is a 

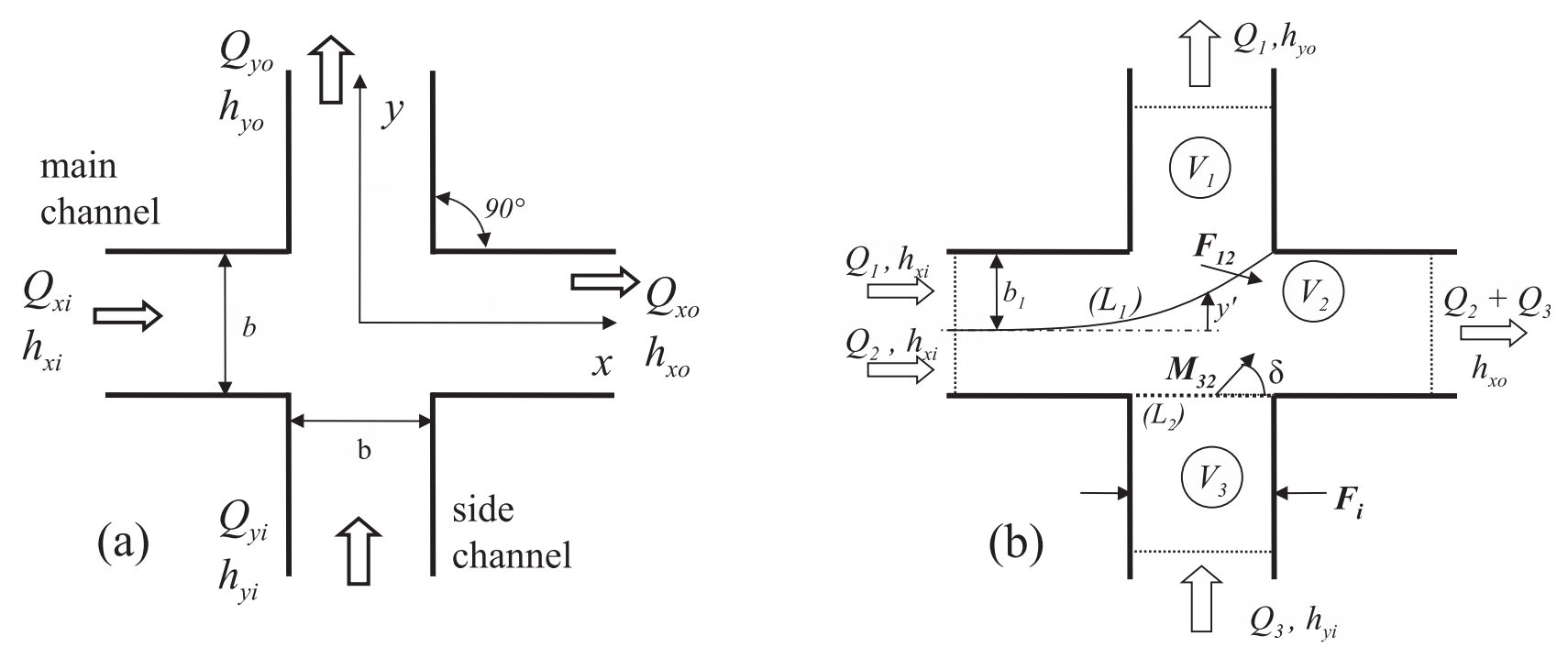

Figure 1. Junction composed of four identical channels intersecting at right angles.

square of side $b$. We denote the acceleration due to gravity by $g$. We define the main, axial channel (and hence the direction $x$ ) as the inlet channel carrying the greatest flow, so the flow in the side inlet channel (direction $y$ ) is always less than or equal to the flow in the main channel. Assuming that the flow can be characterized by a one-dimensional representation, the problem is completely defined by the following eight variables that are the flow rate and the depth:

[9] 1. in the main inlet channel: $Q_{x i}$ and $h_{x i}$,

[10] 2. in the side inlet channel: $Q_{y i}$ and $h_{y i}$,

[11] 3. in the main outlet channel: $Q_{x o}$ and $h_{x o}$,

[12] 4 . in the side outlet channel: $Q_{y o}$ and $h_{y o}$.

[13] The only parameters that are imposed (and are therefore known a priori) are the inlet flow rates $Q_{x i}$ and $Q_{y i}$; we therefore require six equations to close the problem.

[14] The first one is the continuity equation:

$$
Q_{x i}+Q_{y i}=Q_{x o}+Q_{y o}
$$

[15] Following the literature review of section 1.1, we adopt the commonly used assumption of equal depths in the two inlet channels. Measurements performed in some of the configurations studied here confirmed the validity of this assumption to within $\pm 5 \%$, and as far as we are aware this is the only validation of the assumption for a four branch intersection. It is possible to derive a set of model equations that do not rely on this assumption [e.g., Shabayek et al., 2002] but the model is sensitive to the values of various empirical coefficients, which have not been measured for the four branch problem. This assumption yields

$$
h_{x i}=h_{y i} .
$$

[16] Two further equations can be obtained from the boundary conditions at the exits of the two outlet channels, which link the depths to the flow rates. For the experimental conditions used in this work, these relationships are imposed by the weirs that are used to regulate the flow depths in the channels; the equations themselves are provided in section 2 , and we then show how they can be generalized to any boundary conditions likely to be encountered in practical applications. The fifth equation is derived in section 3 by extending the models developed for three branch intersections, based on a momentum balance in the intersection, which relate the flow distribution to the ratio of flow depths. Finally, the sixth equation is derived in section 4, using dimensional analysis and experimental results to obtain an empirical correlation for the flow distribution.

\section{Experimental Arrangements}

\subsection{Experimental Geometry and Measurement Techniques}

[17] The setup consists of an intersection of four identical channels which intersect at right angles [Rivière et al., 2006]. Each channel is made from glass, with a length $L=2$ $\mathrm{m}$ and width $b=0.3 \mathrm{~m}$. The slopes of the four channels can be fixed independently (in this experiment at $0 \%$ ), and each channel can function either as an inlet or an outlet for the intersection. The channels are aligned with the $x$ and $y$ axes; the flow enters through two of the channels. At the inlet to each channel a honeycomb screen straightens and smoothes the flow. Surface waves are eliminated by mean of a sheet of polyurethane floating on the free surface to ensure that it is reasonably smooth. The inlet flow rates $Q_{x i}$ and $Q_{y i}$ can be varied between 0 and $121 \mathrm{~s}^{-1}$, and the conditions are always chosen so that $Q_{x i} \geq Q_{y i}$. The discharges at the inlets and the outlets are measured by four identical electromagnetic flowmeters (Promag 50 from Endress \& Hauser) with an uncertainty of $\pm 0.051 \mathrm{~s}^{-1}$. Water depths are measured using a movable point gauge with an uncertainty of $\pm 0.15 \mathrm{~mm}$, at least in the absence of surface waves.

[18] At a scale of $1 / 24$, the $300 \mathrm{~mm}$ wide channels would represent streets $7.20 \mathrm{~m}$ wide, which is representative of streets in the center of old European towns. In the experiments, the water depth ranges typically from 15 to 120 $\mathrm{mm}$, corresponding to flood depths in the range 0.36 to $2.88 \mathrm{~m}$. This matches values observed during flood events in dense urban areas, such in Nîmes, France, in 1988 Mignot et al. [2006]. 


\subsection{Outlet Control Weirs}

[19] In order to control the flow conditions, PVC channels (length $61 \mathrm{~cm}$ ) fitted with sharp crested weirs were added to the ends of the two exit channels. The weirs are continuously adjustable so that the crest levels $c_{x}$ and $c_{y}$ can be set to any values between 0 and $180 \mathrm{~mm}$. The depths upstream of the weirs ( $h_{x o}$ and $h_{y o}$, respectively) can be calculated from the flow rates in the channels $\left(Q_{x o}\right.$ and $\left.Q_{y o}\right)$ and the stage-discharge relationships for the weirs. For those experiments in which the discharge rate is less than $91 \mathrm{~s}^{-1}$, the Reynolds number $\operatorname{Re}=4 Q / b \nu$ is less than $10^{5}$, and if the discharge rate falls below $1 \mathrm{~s} \mathrm{~s}^{-1}$ then the Weber number becomes less than 10 . It was therefore necessary to calibrate the weirs for the range of operating conditions used in these experiments. The stage-discharge relationship was defined in terms of an "equivalent weir" (see section 2.3 for a full definition of this concept); the depth measured 1.5 widths upstream of the weir is given as a function of the flow rate by the equation

$$
h_{p o}=c_{p}+\left[1+0.793\left(\frac{c_{p}}{b}\right)^{0.731}\right]\left(\frac{Q_{p o}^{2}}{g b^{2}}\right)^{1 / 3}
$$

where $p=x, y$ depending on the channel under consideration. This empirical correlation was obtained from a set of 70 data points, consisting of 10 different flow rates $\left(Q_{p o}=\right.$ $0.2-201 \mathrm{~s}^{-1}$ ) and seven different weir heights $c_{p}=10,20$, $30,50,80,100$, and $120 \mathrm{~mm}$ with the additional constraint that the relationship should yield the critical depth as $c_{p} \rightarrow$ 0 . The maximum difference between the theoretical and the measured depth varied between $-7 \%$ and $3.4 \%$. For flow conditions for which the Reynolds number exceeds $10^{5}$, equation (3) is equivalent to standard stage-discharge relationships such as that given by Rehbock and Böss, cited by Graf [2000]. Such weirs create an upstream flow recirculation that could be avoided using other downstream control devices. Nevertheless, with the length to width ratio of the present channels, the effects of recirculation are expected to be negligible.

\subsection{The Downstream Boundary Conditions, and the Notion of an "Equivalent" Weir}

[20] There are two main reasons for defining the characteristics of the flow in the intersection in terms of the flow at the outlet weirs. First, in most of the existing studies [e.g., Ramamurthy and Satish, 1988], the correlations that are proposed are defined in terms of the depths downstream of the intersection, obtained directly from measurements, or in terms of parameters that are based on them, such as the Froude number. But these downstream depths are not well defined or easy to measure; the stagnation and separation points and the recirculating regions in the intersection all generate surface waves which are the source of uncertainty and error in the depth measurements. The use of a stage-discharge relationship at the outlet of the channel avoids this problem; the flow rate in the channel is known (measured with reasonable precision by an electromagnetic flow meter) and the weir height can be measured precisely, so that the effective water depth is known, using equation (3), whatever the perturbations of the flow introduced by conditions in the intersection.
[21] The second reason for defining the flow in terms of a stage-discharge relationship is that it facilitates the use of dimensional analysis (see section 4.1) to analyze the experimental data; the weir height yields a dimensionless variable which can be varied independently of the flow rate, and this is an advantage over the direct use of the downstream depth.

[22] It could be argued that the use of weir heights to characterize the depth downstream of the intersection is somewhat artificial, since it is unlikely that such a control structure would exist in any real situation, in an urban environment. The exact method for generalizing our results to any boundary condition is developed in section 5 .

\section{Momentum Balance}

\subsection{Control Volumes and Modeling Assumptions}

[23] A momentum balance applied to the flow in the intersection can only provide one additional equation [Taylor, 1944]. The flow leaving the intersection via the side branch is too strongly three-dimensional, principally because of the recirculating region, to be described by a one-dimensional model. As a result, the conservation of momentum can only be used in the $x$ direction. Even then, the equation involves additional unknown terms, resulting from the forces exerted on the flow by the channel walls. This problem has already been studied for intersections of three identical channels at $90^{\circ}$, for both bifurcations [Ramamurthy et al., 1990] and for junctions [Ramamurthy et al., 1988; Gurram et al., 1997]. We will show here that the hypotheses used in those studies to derive the momentum balance also apply to the four branch intersection, and that we can therefore derive a similar equation for this flow.

[24] The momentum balance involves three control volumes, $\left[V_{1}\right],\left[V_{2}\right]$, and $\left[V_{3}\right]$ (Figure $1 b$ ) separated by the left hand surface $\left(L_{1}\right)$ and the entry to the side channel $\left(L_{2}\right)$. In order to simplify the problem we make the following five assumptions (H1-H5):

[25] $\mathrm{H} 1$ : the flow regime is subcritical upstream of the intersection, in both inlet branches.

[26] $\mathrm{H} 2$ : the flow crossing the entry sections $A_{x i}$ and $A_{y i}$ and the exit section $A_{x o}$ can be considered one dimensional, and the pressure is therefore hydrostatic at these sections.

[27] H3: the frictional forces on the channel walls are negligible compared with the pressure forces. It follows from this that the pressure forces are the only forces involved in the $x$ axis momentum balance; we denote these forces as $F_{i}$ acting on the flow in the upstream branch and $F_{o}$ acting on the flow in the downstream branch (Figure 1b).

[28] H4: the water depths in both inlet channels upstream of the intersection are the same (equation (2)).

[29] H5: without loss of generality, the entire inlet flow $Q_{y i}$ leaves via the main outlet channel (in the $x$ direction) while the inlet flow $Q_{x i}$ is divided between the two outlet channels, in the $x$ and $y$ directions. In the case of the opposite configuration, $Q_{y i}$ separates in two and $Q_{x i}$ leaves via a single channel, the results obtained in this paper still apply, with a change of variable, as described at the end of this section.

[30] These assumptions define the control volumes, as shown in Figure 1b, and the flow rates through them are 
related to the flow rates that characterize the problem by the following equations:

$$
Q_{x i}=Q_{1}+Q_{2} ; Q_{y i}=Q_{3} ; Q_{y o}=Q_{1} ; Q_{x o}=Q_{2}+Q_{3} .
$$

[31] A momentum balance for each control volume then involves the interactions between the control volumes, which can be reduced to the pressure force $F_{12}$ exerted by $\left[V_{1}\right]$ on $\left[V_{2}\right]$ and the momentum flux $M_{32}$ leaving $\left[V_{3}\right]$ and entering $\left[V_{2}\right]$. Projected onto the $x$ axis, the equation of conservation of momentum for the control volume $\left[V_{2}\right]$ can be written

$$
\begin{aligned}
\rho \beta_{x o} & \frac{\left(Q_{2}+Q_{3}\right)^{2}}{b h_{x o}}-\rho \beta_{x i} \frac{Q_{2}^{2}}{b h_{x i}}-M_{x 32} \\
= & F_{x 12}+\rho g \frac{h_{x i}^{2}}{2} b \frac{Q_{2}}{Q_{1}+Q_{2}}-\rho g \frac{h_{x o}^{2}}{2} b,
\end{aligned}
$$

where $\beta_{x i}$ and $\beta_{x o}$ are Boussinesq momentum coefficients. The expression for the force $F_{x 12}$ can be obtained in the way described by Ramamurthy et al. [1990] for a three branch bifurcation. Using the variables of the present paper, this can be written

$$
F_{x 12}=\frac{1}{2} \rho g b \frac{Q_{y o}}{Q_{x i}}\left[h_{x i}^{2}+\frac{Q_{x i}^{2}}{3 g b^{2} h_{x i}}+\frac{Q_{x i}^{4}}{20 g^{2} b^{4} h_{x i}^{4}}\right] .
$$

[32] The term $M_{x 32}$ in equation (5) represents a momentum flux in the $x$ direction that enters $\left[V_{2}\right]$ via the flow through the upstream side channel. This occurs because the free surface level in the side channel, just upstream of the intersection, is not horizontal; the difference in surface elevation across the channel generates a pressure force $F_{i}$, and the effect of this force can be modeled as an additional momentum flux along the main channel, as has been suggested by several authors [e.g., Ramamurthy et al., 1988; Gurram et al., 1997; Hsu et al., 1998].

[33] The form of the momentum flux term depends on the geometry of the intersection, and the flow conditions; the closest configuration to the four branch intersection investigated here is the three branch junction studied by Ramamurthy et al. [1988], which also consisted of identical channels intersecting at right angles. For that case, they obtained the following expression for the momentum flux:

$$
\begin{aligned}
M_{x 32} & =\rho \frac{Q_{3}^{2}}{b h_{y i}} \frac{1}{\tan \delta}=\rho \frac{Q_{y i}^{2}}{b h_{y i}} \frac{1}{\tan \delta} \\
\text { with } \tan \delta & =\frac{Q_{3}}{Q_{2}}=\frac{Q_{y i}}{Q_{x o}-Q_{y i}} .
\end{aligned}
$$

[34] Expressions for the force $F_{x 12}$ (equation (6)) and the momentum flux $M_{x 32}$ (equation (7)) are substituted into the momentum balance equation (5). The latter is rewritten in dimensionless form, using the dimensionless variables

$$
\begin{gathered}
R_{q}=\frac{Q_{y o}}{Q_{x i}}, \quad R_{h}=\frac{h_{x i}}{h_{x o}}, \quad R_{q i}=\frac{Q_{y i}}{Q_{x i}}, \\
R_{x o}=\frac{h_{x o}}{b}, \quad R_{g}=\frac{Q_{x i}}{b^{2} \sqrt{g b}} .
\end{gathered}
$$

[35] If the Boussinesq coefficients in equation (5) are both assumed equal to $1\left(\beta_{x o}=\beta_{x i}=1\right)$, as has been assumed in previous studies, then the dimensionless momentum balance equation becomes

$$
\begin{aligned}
& \frac{-R_{x o}^{3} R_{h}^{5}}{2 R_{g}^{2}}+\left(\frac{R_{x o}^{3}}{2 R_{g}^{2}}+1+2 R_{q i}-2 R_{q}+R_{q i}^{2}-2 R_{q i} R_{q}+R_{q}^{2}\right) R_{h}^{3} \\
& +\left(\frac{5}{6} R_{q}-1+R_{q i} R_{q}-R_{q i}\right) R_{h}^{2}-\frac{R_{q} R_{g}^{2}}{40 R_{h} R_{x o}^{3}}=0 .
\end{aligned}
$$

[36] There is no analytical solution for equation (8), but it can be solved by iteration. The parameter $R_{x o}$ depends on the downstream depth $h_{x o}$, which can be obtained from the flow rate $Q_{x o}$ and the stage-discharge relationship for the weir (see section 2.3). From this equation it is possible to compute $R_{h}$, and hence $h_{x i}$, as a function of the flow distribution. As a check on this equation, we can evaluate it with $R_{q i}=0$; as required, the resulting equation is then identical to that proposed by Ramamurthy et al. [1990] for a simple bifurcation, taking into account that they have used a different definition for the parameter $R_{q}$. This equation was derived for the condition where the main upstream discharge separates in two $\left(Q_{y i}<Q_{x o}\right.$, hypothesis H5). It is equally valid when the minor upstream discharge separates in two $\left(Q_{y i}>Q_{x o}\right)$ provided that the control volumes are modified, with the result that the variables $x$ and $y$ are permuted. In the following section we will test the validity of the assumptions used to derive the equation, using experimental observations and data.

\subsection{Comparison with Experiments}

[37] The theoretical values of the depth ratio $R_{h}$, given by the solution to equation (8) are plotted in Figure 2 as a function of the experimental values $\left(h_{x i}\right.$ measured onewidth upstream of the intersection and $h_{x o}$ one width and a half upstream of the weir). The agreement is generally satisfactory, with the values agreeing to within $\pm 4 \%$ over the entire range of values of $R_{h}$ used in the experiments. On the basis of these measurements it seems reasonable to conclude that the momentum balance equation (8) provides a valid model for the flow in the intersection. This equation could be rewritten, using equations (1)-(3), to provide an equation linking two of the unknown quantities- $h_{x o}$ and $Q_{x o}$, say, or $h_{y o}$ and $Q_{y o}$-in terms of the other imposed conditions. So we only require one additional equation involving the same two unknown variables to close the system. An energy balance could provide such an equation, and it could be rewritten in the same way as for equation (8), but unfortunately, as indicated previously, it would be unlikely to yield accurate results, because of the uncertainty concerning the energy losses in the intersection. So instead we have had to resort to an empirical correlation, derived from our data. It would not be possible to obtain such a correlation simply by plotting one of the unknown variables as a function of the other, because of the large number of independent parameters in the problem, which are likely to feature in the eventual correlation; we have instead adopted an approach based on dimensional analysis of the independent parameters in the experiments. 


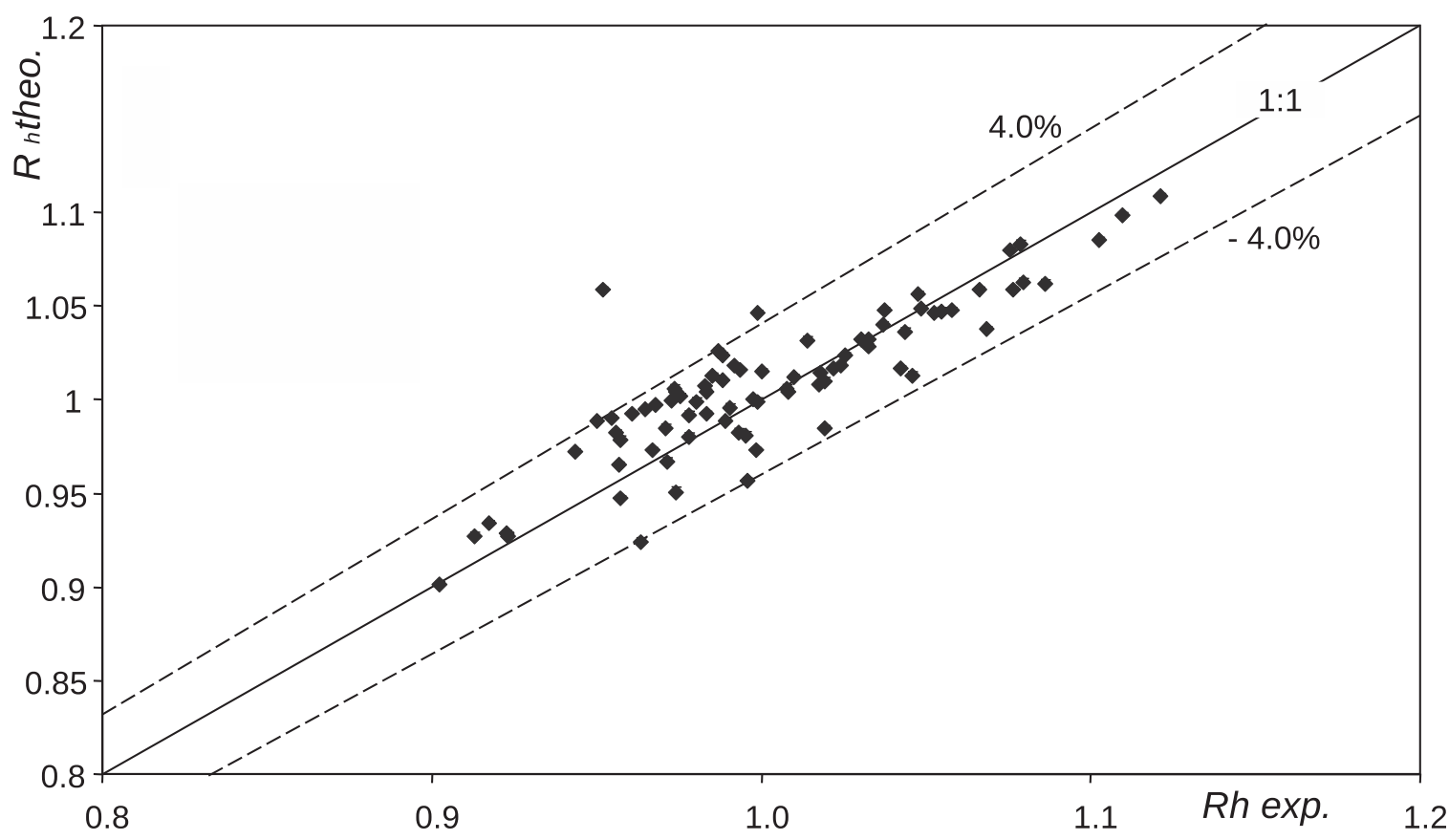

Figure 2. Comparison of the theoretical and measured values of $R_{h}$.

\section{An Empirical Correlation Derived Using Dimensional Analysis}

\subsection{Dimensional Analysis}

[38] The problem is fully characterized by 12 parametersthe four flow rates $Q_{x i}, Q_{y i}, Q_{x o}$, and $Q_{y o}$, the four depths $h_{x i}, h_{y i}, h_{x o}$, and $h_{y o}$, and four parameters that characterize the installation itself: the weir heights $c_{x}$ and $c_{y}$, the channel width $b$, and the gravitational acceleration $g$.

[39] The preceding analysis has already yielded five equations linking these variables and we can now use these to simplify the problem somewhat; the seven independent variables that define the problem are $b, g, Q_{x i}, Q_{y i}, c_{x}, c_{y}$, and $Q_{y o}$.

[40] If we take $b$ for our length scale and $b^{3} / Q_{x i}$ for our time scale, dimensional analysis yields

$$
\Phi\left(\frac{Q_{y o}}{Q_{x i}}, \frac{Q_{y i}}{Q_{x i}}, \frac{c_{x}}{b}, \frac{c_{y}}{b}, \frac{Q_{x i}}{b^{2} \sqrt{g b}}\right)=0
$$

Here we have neglected the effects of viscosity and surface tension (see section 2) which would have resulted in two additional dimensionless numbers, the Reynolds number and the Weber number.

[41] By rearranging these dimensionless parameters we can write the flow distribution ratio as

$$
\frac{Q_{y o}}{Q_{x i}}=F\left(\frac{Q_{y i}}{Q_{x i}}, \frac{c_{y}}{c_{x}}, \frac{c_{x}}{b}, \frac{Q_{x i}}{b^{2} \sqrt{g b}}\right)
$$

which is written, for simplicity:

$$
R_{q}=F\left(R_{q i}, R_{c}, R_{b}, R_{g}\right)
$$

[42] Equation (11) actually represents a surface in fivedimensional space. A detailed description of this surface would require a very large number of experimental measurements, $10^{4}$, if each dimensionless parameter is discretised into 10 values, and this is not feasible. However a preliminary study [Rivière et al., 2006] showed a simple linear dependency of $R_{q}$ on some of the other parameters; in certain conditions (e.g., $\left.R_{c}=1\right) R_{q}$ was even independent of some of the parameters. Guided by those preliminary results, we have constructed an empirical correlation for equation (14) using the projection of the surface onto the different planes $\left(R_{q}, \Pi\right)$ where $\Pi$ is one of the parameters, the three others being held constant.

\subsection{Empirical Correlation}

[43] This preliminary study showed that if all the other parameters are held constant, $R_{q}$ varies linearly with $R_{q i}$. This can be seen in Figure $3 \mathrm{a}$, where we have plotted the results for data sets 0 and 2 . For any given value of $R_{c}$ the data points agree well with the straight line obtained by linear regression; both the slope and the intercept depend on $R_{c}$. In Figure $4 \mathrm{a}, R_{q}$ has been plotted as a function of $R_{q i}$ for different values of $R_{b}$, the other parameters being constant; this shows that the intercept depends on $R_{b}$, but the slope does not. The same procedure was performed to characterize the influence of $R_{g}$ and show that the intercept depends on $R_{g}$, but the slope does not. Moreover, for the particular case $R_{c}=1$, the relation between $R_{q}$ and $R_{q i}$ is not affected by the other parameters [Rivière et al., 2006] as shown in Figure 3a. Therefore the discharge distribution will depend on the different parameters in the following way:

$$
R_{q}=S\left(R_{c}\right) \times R_{q i}+B+\left(1-R_{c}\right) \times C\left(R_{c}, R_{b}, R_{g}\right) .
$$

[44] To examine the influence of $\left(R_{c}, R_{b}, R_{g}\right)$ on the intercept, $R_{q}$ is plotted successively as a function of each of these parameters, $R_{q i}$ being constant. 

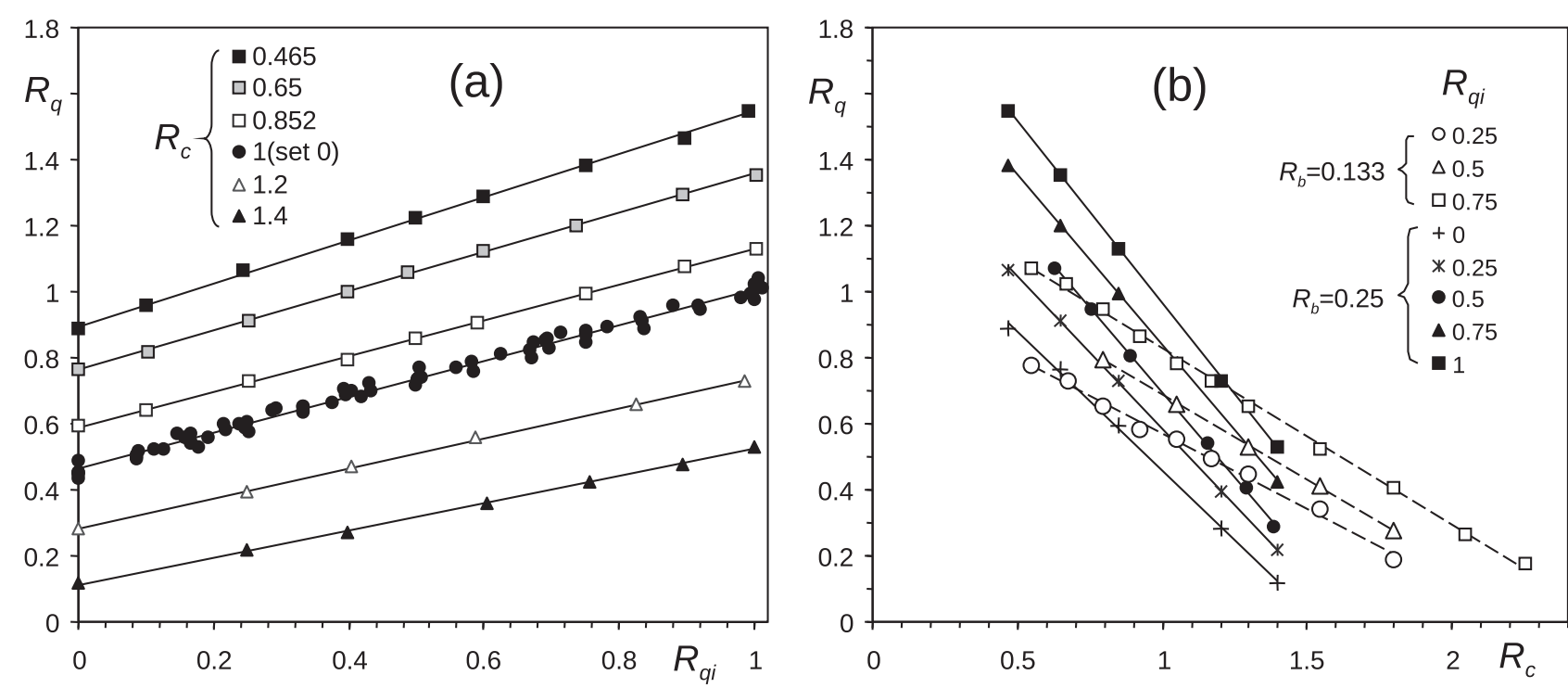

Figure 3. (a) $R_{q}$ as a function of $R_{q i}$ for constant values of $R_{c}$ with $R_{b}=0.25$ and $R_{g}=0.0389$ and (b) as a function of $R_{c}$ for constant values of $R_{b}$ with $R_{g}=0.0389$.

[45] Figure 3b shows that $R_{q}$ depends linearly on $R_{c}$, and the intercept must take the form $C=C\left(R_{b}, R_{\mathrm{g}}\right)$. The slope is also necessarily a linear function of $R_{c}: S=A R_{c}+A_{0}$. As we know that $R_{q}=1$ when $R_{c}=1$ and $R_{q i}=1, R_{q}$ can be written in the following form:

$$
R_{q}=\left[1-A-B+A R_{c}\right] \times R_{q i}+B+\left(1-R_{c}\right) \times C\left(R_{b}, R_{g}\right) .
$$

[46] Figure 4b shows that $R_{q}$ depends linearly on $R_{b}$. Finally, considering $R_{g}^{-1}$, the dependency (not presented here) is again linear.

[47] The final correlation providing the discharge distribution $R_{q}$ reads

$$
\begin{aligned}
R_{q}= & {\left[1-A-B+A R_{c}\right] \times R_{q i}+B } \\
& +D \times\left(R_{c}-1\right)\left(R_{b}+E\right)\left(R_{g}^{-1}+F\right)
\end{aligned}
$$

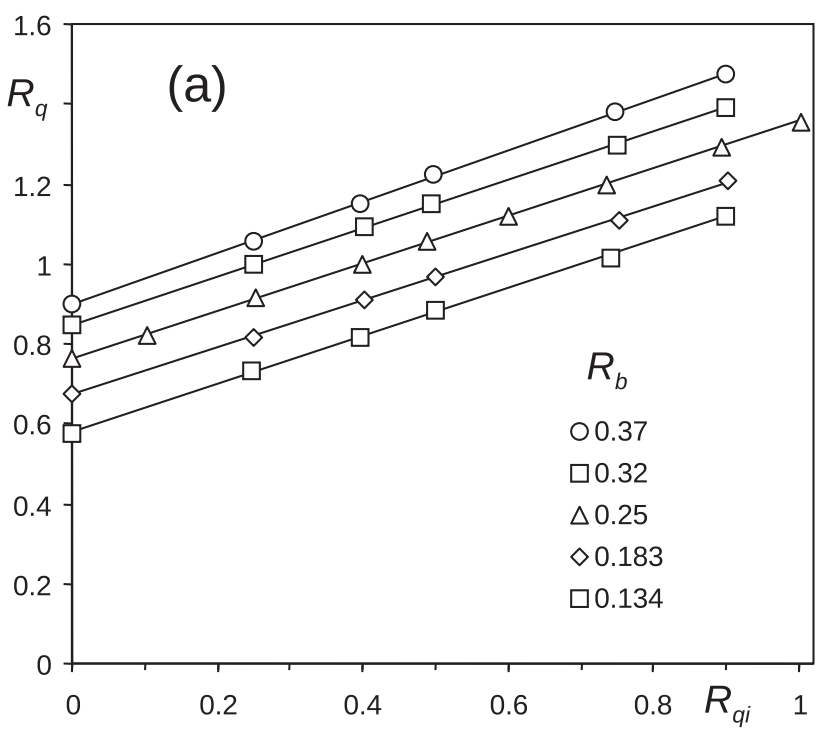

with $A=-0.141, B=0.456, D=-0.113, E=-0.030$, $F=9.797$. These values of constants $A, B, D, E$. and $F$ have been estimated using the 220 data points obtained in these experiments and in the experiments reported by Rivière et al. [2006]. We used a method of least squares, although other methods are available, such as for instance principal component analysis (PCA) or partial least square (PLS) [Ramamurthy et al., 2006]. The correlation coefficient is 0.9968 and the result is plotted in Figure 5. The data points agree well with the empirical correlation over the full range of experimental conditions, with an error that rarely exceeds $\pm 5 \%$. This correlation was derived for a fairly wide range of values for the four dimensionless parameters:

$$
\begin{aligned}
0< & R_{q i}<1 ; 0.4<R_{c}<2.5 ; 0.1<R_{b}<0.37 ; 0.0226 \\
& <R_{g}<0.0651
\end{aligned}
$$

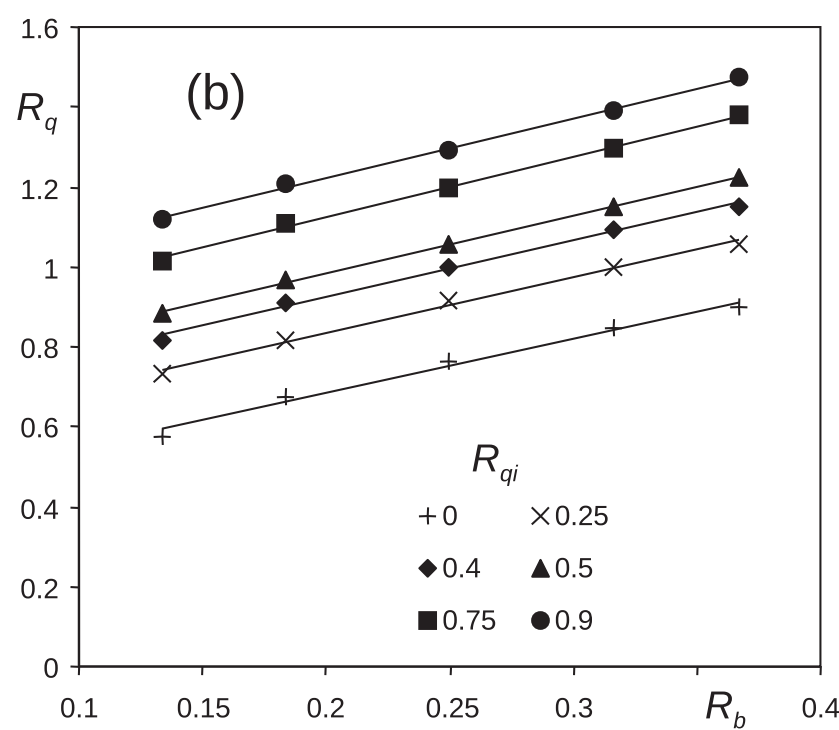

Figure 4. (a) $R_{q}$ as a function of $R_{q i}$ for constant values of $R_{b}$ and (b) as a function of $R_{b}$ for constant values of $R_{q i}$ (with $R_{g}=0.0389$ and $R_{c}=0.65$ ). 


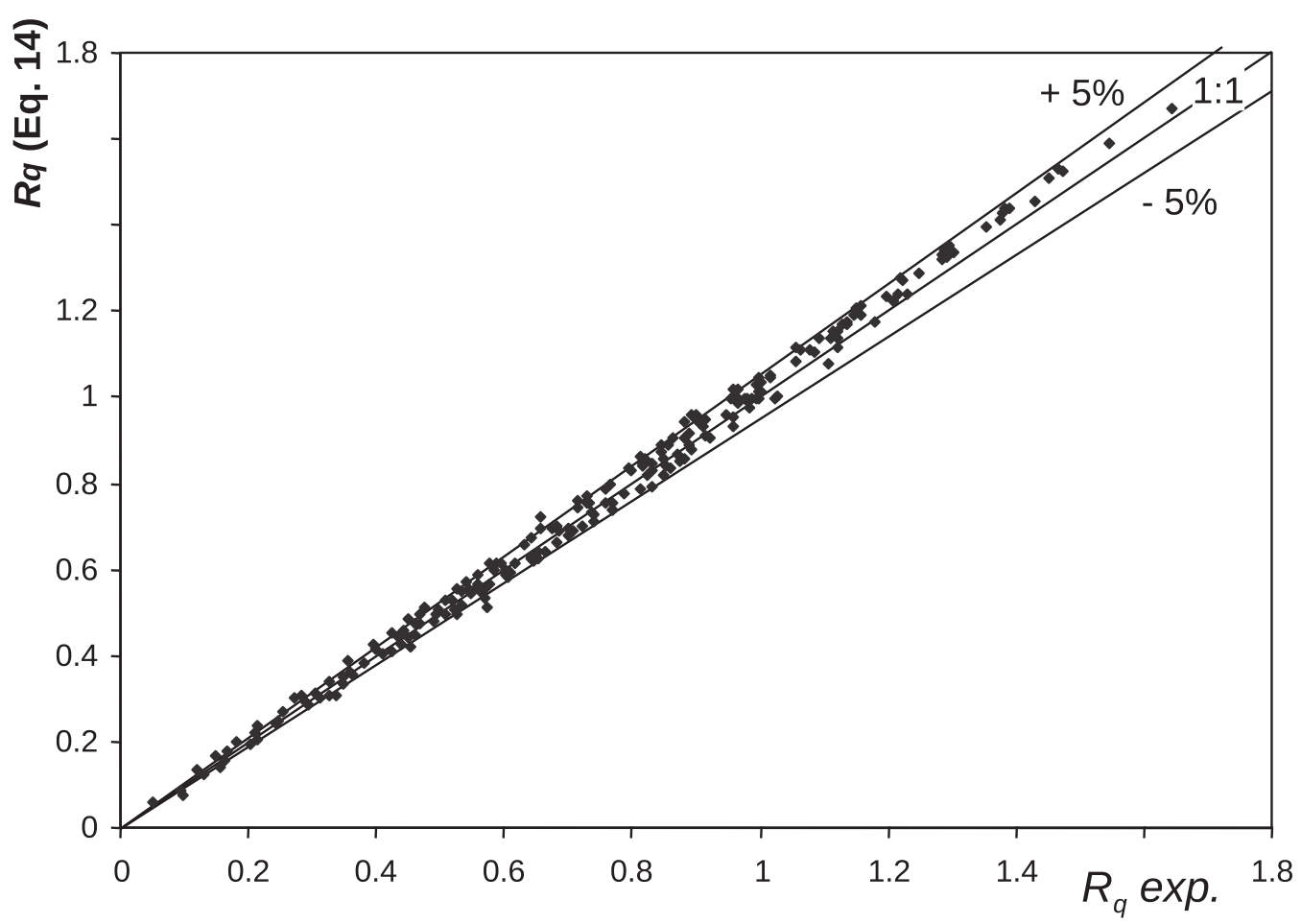

Figure 5. Comparison of the theoretical values of $R_{q}$ given by the empirical correlation (14) with the experimental values of $R_{q}$.

which must nevertheless be taken as delimiting the range of validity of the correlation (equation (14)), in the absence of any data outside these limits. It should also be remembered that the flows are strongly turbulent (so the Reynolds number does not figure as a dimensionless parameter) and we have neglected the influence of surface tension, so the Weber number does not appear among the dimensionless parameters either.

[48] The empirical correlation (14) therefore represents the sixth and final equation necessary to close the model.

\section{Application of the Model}

[49] The six equations that constitute the model [(1), (2), and (3) for $p=x$; (3) for $p=y$; and (8) and (14)] can be used to compute the flow distribution and the flow depths in the outlet channels, given the flow rates in the two inlet channels. If we assume that the equivalent weir heights $c_{x}$ and $c_{y}$ are known, then the empirical correlation (14) provides the outlet flow distribution $\left(R_{q}=Q_{y o} / Q_{x i}\right)$ as a function of the inlet flow distribution $\left(R_{q i}=Q_{y i} / Q_{x i}\right)$, which is known, and the other dimensionless parameters $\left(R_{c}, R_{b}\right.$, and $R_{g}$ ) which characterize the geometry of the configuration. The depths in the downstream channels $\left(h_{x o}\right.$ and $h_{y o}$ can then be obtained from the flow rates in the channels $\left(Q_{x o}\right.$ and $\left.Q_{y o}\right)$ and the stage-discharge relations for the channel controls. Finally, the depths in the inlet channels ( $h_{x i}$ and $\left.h_{y i}\right)$ can be calculated from the inlet flow rates $\left(Q_{x i}\right.$ and $Q_{y i}$ ) and equation (8).

[50] The model can therefore be used for any configuration for which the equivalent downstream weirs are known. Of course, in practice, the flows in the outlet channels will not be controlled by weirs, but as discussed earlier, it will always be possible to define an equivalent weir for the control structure, provided the stage-discharge relationship for the structure is known. The problem is that, in order to compute this equivalent weir, it is necessary to know the discharge in the channel, and this can only be calculated (from equation (14)) if the equivalent weir is already known! The only way to solve this is to assume an initial value for $R_{q}$, compute the corresponding depths $h_{x o}$ and $h_{y o}$ from the stage-discharge relationships, and deduce the equivalent heights $c_{x}$ and $c_{y}$ from equation (3). All the variables needed to solve equation (14) are then available, and a new value of $R_{q}$ can be calculated using this equation. This will not, in general, be the same as the value assumed initially, and so it is necessary to iterate this procedure, adjusting the value of $R_{q}$ each time, until the system converges.

[51] Two practical problems can arise in the application of this iterative procedure. The first occurs when the downstream control leads to the blockage of one of the channels, and this can occur even if all the dimensionless parameters remain within the domain of validity given in this paper. At high values of $R_{c}$, for example, the outlet discharge in the side channel can fall to zero, in which case the value of $R_{q}$ given by equation (14) will become negative; the best solution to this problem is then to set $R_{q}=Q_{y o}=0$. A similar problem arises when $R_{c}$ becomes very small, and $R_{q} \geq$ $\left(1+R_{q i}\right)$, indicating that all the flow leaves through the side outlet channel, so that $R_{\mathrm{q}}=\left(1+R_{q i}\right)$ and $Q_{x o}=0$.

[52] The second problem occurs if the Froude number in one of the channels exceeds 1 . Indeed, the equations all assume that the flow remains subcritical everywhere, and are not valid for the supercritical regime. The Froude number of the flow in each channel can be calculated from a combination of the six dimensionless parameters $\left(R_{q}, R_{q i}\right.$, 
$R_{c}, R_{b}, R_{g}, R_{h}$ ) and it is possible for the computed flow in one or other of the downstream channels to become supercritical. In practice, this will occur in one of the regions where the flow contracts because of the presence of a recirculating region. In these flow configurations equation (14) is no longer applicable, but their appearance can be predicted by deriving $\mathrm{Fr}_{y o}$; results in the literature suggest they will appear for $F r_{y o} \gtrsim 0.34$ (1/3 for Hager [1999]; 0.35 for Ramamurthy and Satish [1988]).

[53] Two types of critical section can exist in the axial direction. The first forms just downstream of the intersection, at the contraction created by the combination of the two incoming flows; this has been observed in three branch junctions, but never in the experimental conditions studied here. A second type of critical section can form just upstream of the intersection if $R_{q i}=0$ and if the downstream control in the main downstream channel is weaker than in the side channel. This is caused by the acceleration of the incoming flow in the main channel [Law and Reynolds, 1966; El Kadi Abderrezzak et al., 2011]. This type of transition was observed in some of the experiments reported here, but only for $R_{q i}=0$ and $R_{\mathrm{c}}>2$.

\section{Conclusion}

[54] The flow distribution in a four branch intersection has been studied experimentally for subcritical flow in all four channels. The water depths in the two outlet channels were controlled by weirs at the downstream end of the channels, and the heights of these weirs were introduced as control parameters for the problem. We have developed an empirical correlation for the distribution of the flow in the channels leaving the intersection, based on the results of 220 experiments over a wide range of conditions. This correlation depends only on the incoming flows and the heights of the downstream weirs. We have shown that it is possible to define an equivalent height for any downstream control structure, provided that the stage-discharge relationship for the structure is known, and that it is therefore possible to use this correlation for any situation which corresponds to the range of conditions studied in these experiments. The depths in the channels upstream of the intersection can be computed from other relationships, derived from a physical analysis of the flow.

[55] The correlation also provides a fairly compact and accurate summary of a large number of experimental results, so it can be used to calibrate or validate numerical simulations, which could then be applied to other, more complicated geometries.

[56] Despite the success of the correlation in reproducing the entire data set with a relatively simple formula, further work is still required. First, it would be useful to compare the correlation with data sets that fall within the domain of validity, but which were not used in the derivation of the correlation. This would test the ability of the formula to interpolate within the data sets. It would then be interesting to compare the correlation with data for conditions outside the domain of validity, to test its capacity to extrapolate from the original data sets.

[57] In some of the experiments the flow underwent a transition from subcritical to supercritical, and we have identified three different mechanisms that could cause this, depending notably on the downstream conditions: appearance of a critical section in the downstream branch or in the main downstream channel, or critical regime at the entrance of the intersection. The results presented in this paper are only valid if the flow is subcritical everywhere in the intersection, and the existence of several different transition mechanisms indicates that the transcritical regime requires a separate study to complement and complete the results presented here and extend the domain of validity of the model to those cases in which the flow is blocked by the presence of a critical section. This would also provide a link with other similar studies of supercritical flow in intersections.

[58] Acknowledgments. The research was funded by the French CNRS (SPI-ACI jeune chercheur "Hydraulique en milieu urbain", 2002; INSU: ECCO-PNRH 2003, project 31) and the French ANR $\left(\mathrm{Hy}^{2}\right.$ Ville project, grant ANR-05-ECCO-016; Rives project, grant ANR-05-PGCU-004).

\section{References}

Barkdoll, B. B., B. L. Hagen, and A. J. Odgaard (1998), Experimental comparison of dividing open-channel with duct flow in T-Junction, $J$. Hydraul. Eng., 124(1), 92-95.

Best, J. L., and I. Reid (1984), Separation zone at open-channel junctions, J. Hydraul. Eng., 110(11), 1588-1594.

Calenda, G., L. Calvani, and C. P. Mancini (2003), Simulation of the Great Flood of December 1870 in Rome, Water Mar. Eng., 156(4), 305-312.

El Kadi Abderrezzak, K., A. Paquier, and E. Mignot (2009), Modelling flash flood propagation in urban areas using a two-dimensional numerical model, Nat. Hazards, 50(3), 433-460.

El Kadi Abderrezzak, K., L. Lewicki, A. Paquier, N. Rivière, and G. Travin (2011), Division of critical flow at three-branch open-channel intersection, J. Hydraul. Res., 49(2), 231-238.

Gisonni, C., and W. H. Hager (2002), Supercritical flow in the $90^{\circ}$ junction manhole, Urban Water, 4, 363-372.

Graf, W. H. (2000), Hydraulique Fluviale, Presses Polytechniques et Universitaires Romandes, Lausanne, Switzerland (in French).

Gurram, S. K., K. S. Karki, and W. H. Hager (1997), Subcritical junction flow, J. Hydraul. Eng., 123(5), 447-455.

Hager, W. H. (1989), Transitional flow in channel junctions, J. Hydraul. Eng., 115(2), 243-259.

Hager, W. H. (1992), Discussion of "Dividing flow in open channels" by A. S. Ramamurthy, D. M. Tran, and L. B. Carballada, J. Hydraul. Eng., $118(4), 634-637$.

Hager, W. H. (1999), Wastewater Hydraulics, Springer, Berlin.

Haider, S., A. Paquier, R. Morel, and J.-Y. Champagne (2003), Urban flood modeling using computational fluid dynamics, Water Mar. Eng., 156(72), 129-135.

Hsu, C. C., W. J. Lee, and C. H. Chang (1998), Subcritical open-channel junction flow, J. Hydraul. Eng., 124(8), 847-855.

Hsu, M. H., S. H. Chen, and T. S. Chang (2002), Dynamic inundation simulation of storm water interaction between sewer system and overland flows, J. Chin. Inst. Eng., 25(2), 171-177.

Huang, J., L. J. Weber, and Y. G. Lai (2002), Three-dimensional numerical study of flows in open-channel junctions, J. Hydraul. Eng., 128(3), 268-280.

Inoue, K., K. Kawaike, and H. Hayashi (2000), Numerical simulation models of inundation flow in urban area, J. Hydrosci. Hydraul. Eng., 18(1), 119-126.

Kesserwani, G., M. Abdallah, J. Vazquez, N. Rivière, Q. Liang, G. Travin, and R. Mosé (2010), New approach for predicting flow bifurcation at right-angled open-channel junction, J. Hydraul. Eng., 136(9), 662-68.

Krishnappa, G., and K. Seetharamiah (1963), A new method for predicting the flow in a $90^{\circ}$ branch channel, La Houille Blanche, 18(7), 775-778.

Lakshmana-Rao, N. S., and K. Sridharan (1967), Division of flow in open channels, Irrig. Power, 24(4), 393-407.

Law, S. W., and A. J. Reynolds (1966), Dividing flow in open channel, $J$. Hydraul. Div., 92(HY2), 207-231.

Li, C. W., and C. Zeng (2009a), Numerical modelling of flow divisions at open channel junctions with or without vegetation, Adv. Water Res., $32(1), 49-60$.

Li, C. W., and C. Zeng (2009b), Flow division at a channel crossing with subcritical or supercritical flow, Int. J. Numer. Meth. Fluids, 62(1), 56-73. 
Lipeme-Kouyi, G., N. Rivière, V. Vidalat, A. Becquet, B. Chocat, and V. Guinot (2010), Urban flooding: 1D modelling of the distribution of the discharges through cross-road intersections accounting for energy losses, Water Sci. Technol., 61(8), 2021-2028.

Mignot, E., A. Paquier, and S. Haider (2006), Modeling floods in a dense urban area using 2D shallow water equations, J. Hydrol., 327(1-2), 186-199.

Mignot, E., A. Paquier, and N. Rivière (2008a), Experimental and numerical modeling of symmetrical four-branch supercritical cross junction flow, J. Hydraul. Res., 46(6), 723-738.

Mignot, E., N. Rivière, R. J. Perkins, and A. Paquier (2008b), Flow patterns in a four branches junction with supercritical flow, J. Hydraul. Eng., 134(6), 701-713.

Mignot, E., N. Rivière, A. Paquier, and R. J. Perkins (2011), Hydraulic models of the flow distribution in a four branch open channel junction with supercritical flow, J. Hydraul. Eng., 137(3), 289-299.

Nania, L. S., M. Gomez, and J. Dolz (2004), Experimental study of the dividing flow in steep street crossings, J. Hydraul. Res., 42(4), 406-412.

Neary, V. S., and A. J. Odgaard (1993), Three-dimensional flow structure at open-channel diversions, J. Hydraul. Eng., 119(11), 1223-1230.

Neary, V. S., F. Sotiropoulos, and A. J. Odgaard (1999), Three-dimensional numerical model of lateral-intake inflows, J. Hydraul. Eng., 125(2), $126-140$.

Nougaro, J., and P. Boyer (1974), Sur la séparation des eaux dans les dérivations de canaux à section rectangulaire, La Houille Blanche, 29(3), 199-203 (in French).

Nougaro, J., P. Boyer, and J. Claria (1975), Comportement d'une déviation de canaux lorsque les biefs aval sont pourvus de retenues, La Houille Blanche, 30(4), 267-273 (in French).

Rajaratnam, N. (1962), The constant velocity concept for supercritical branch channel flow, Irrig. Power, 19(1), 17-21.

Ramamurthy, A. S., and M. G. Satish (1988), Division of flow in short open channel branches, J. Hydraul. Eng., 114(4), 428-438.

Ramamurthy, A. S., L. B. Carballada, and D. M. Tran (1988), Combining open channel flow at right angled junctions, J. Hydraul. Eng., 114(12), 1449-1460.
Ramamurthy, A. S., D. M. Tran, and L. B. Carballada (1990), Dividing flow in open channels, J. Hydraul. Eng., 116(3), 449-455.

Ramamurthy, A. S., J. Qu, and D. Vo (2006), Nonlinear PLS method for side weir flows, J. Irrig. Drain. Eng., 132, 486-489.

Ramamurthy, A. S., J. Qu, and D. Vo (2007), Numerical and experimental study of dividing open-channel flows, J. Hydraul. Eng., 133(10), 1135-1144.

Rivière, N., and R. J. Perkins (2004), Supercritical flow in channel intersections, Proceedings of the 2nd International Conference on Fluvial Hydraulics, Grecco, Carravetta, and Della Morte, Eds, River Flow 2004, Napoli, Italy, 23-25 June 2004, 1073-1077.

Rivière, N., R. J. Perkins, B. Chocat, and A. Lecus (2006), Flooding flows in city crossroads: 1D modelling and prediction, Water Sci. Technol., 54(6-7), 75-82.

Shabayek, S., P. Steffler, and F. Hicks (2002), Dynamic model for subcritical combining flows in channel junctions, J. Hydraul. Eng., 128(9), 821-828.

Sridharan, K., and N. S. Lakshmana-Rao (1966), Division and combination of flow in open channels, J. Inst. Eng., 46(7), 337-356.

Taylor, E. H. (1944), Flow characteristics at rectangular open-channel junctions, ASCE Trans., 109, 893-902.

Webber, N. B., and C. A. Greated (1966), An investigation of flow behaviour at the junction of rectangular channels, Proc. Inst. Civ. Eng., 34, 312-334.

Weber, L. J., E. D. Schumate, and N. Mawer (2001), Experiments on flow at a $90^{\circ}$ open-channel junction, J. Hydraul. Eng., 127(5), 340-350.

R. J. Perkins and G. Travin, Laboratoire de Mécanique des Fluides et d'Acoustique (LMFA), CNRS UMR5509, Université de Lyon, École Centrale de Lyon, 36 av. G. de Collongue, F-69134, Ecully, France.

N. Rivière, Laboratoire de Mécanique des Fluides et d'Acoustique (LMFA), CNRS UMR5509, Université de Lyon, INSA de Lyon, Bât Jacquard, 20 av. A. Einstein, F-69621, Villeurbanne, France. (nicolas.riviere@ insa-lyon.fr) 